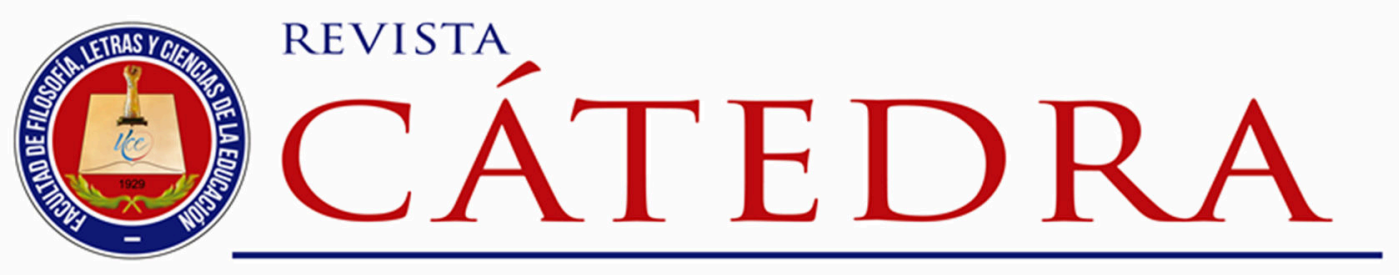

\title{
Sistemas de Aseguramiento de la Calidad: herramienta para la mejora continua de los programas formativos, centros y universidad
}

\author{
Quality Assurance Systems: a tool for the continuous \\ improvement of training programs, centers and \\ universities
}

\author{
Gloria Zaballa-Pérez \\ Universidad de Deusto, Bilbao, España \\ gloria.zaballa@deusto.es \\ https://orcid.org/0000-0002-6609-5968
}

(Recibido: 18/11/2021; Aceptado: 22/11/2021; Versión final recibida: 15/12/2021)

Cita del artículo: Zaballa-Pérez, G. (2022). Sistemas de Aseguramiento de la Calidad: herramienta para la mejora continua de los programas formativos, centros y universidad. Revista Cátedra, 5(1), 58-70.

\section{Resumen}

La Universidad de Deusto tiene definido e implantado en sus facultades un Sistema de Aseguramiento de la Calidad que abarca todas las titulaciones de grado, máster y doctorado, así como la gestión de las actividades que se desarrollan en todas las facultades en su conjunto. El Sistema de Aseguramiento de la Calidad se implanta a través de la sistematización de la metodología de mejora continua, mediante la medición y análisis de la satisfacción de los grupos de interés y de los resultados de sus procesos. Para ampliar el alcance del sistema, se ha definido un Sistema de Gestión de Calidad Global para lograr la satisfacción de los estudiantes, desde su toma de contacto con el mundo universitario, hasta su inserción como egresados en el mercado laboral. Dicho sistema está basado en la filosofía de la gestión por procesos. El Sistema de Gestión de Calidad Global se despliega en los Centros, las Áreas Funcionales y los Servicios implicados en la vida de la comunidad universitaria. El sistema incluye la Acreditación Institucional de las Facultades y las Certificaciones de Calidad de acuerdo a la norma UNE-EN ISO 9001:2015, en las siguientes Áreas Funcionales y Servicios: Nuevos Estudiantes, Biblioteca, Colegio Mayor y Servicio de empleo. 


\section{Palabras clave}

Calidad, herramienta, mejora, sistemática, sostenibilidad.

\section{Abstract}

The University of Deusto has defined and implemented in its faculties a Quality Assurance System that covers all undergraduate, master's and doctoral degrees, as well as the management of the activities carried out in all faculties as a whole. The Quality Assurance System is implemented through the systematization of the continuous improvement methodology, by measuring and analyzing the satisfaction of stakeholders and the results of its processes. To broaden the scope of the system, a Global Quality Management System has been defined to achieve student satisfaction, from their first contact with the university world, to their insertion as graduates in the labor market. This system is based on the philosophy of process management. The Global Quality Management System is deployed in the Centers, Functional Areas and Services involved in the life of the university community. The system includes the Institutional Accreditation of the Faculties and the Quality Certifications according to the UNE-EN ISO 9001:2015 standard, in the following Functional Areas and Services: New Students, Library, Hall of Residence and Employment Service.

\section{Keywords}

Quality, tool, systematic, improvement, sustainability.

\section{Introducción}

La Universidad de Deusto tiene definido e implantado desde el año 2007, un Sistema de Aseguramiento de la Calidad (SAC) para las titulaciones de grado, máster y doctorado, conforme a las directrices del programa AUDIT definidas por ANECA. Este sistema está sometido a un proceso de mejora continua a través de la medición y el análisis de la satisfacción de los grupos de interés, el análisis de los resultados de sus procesos, de las auditorías y su revisión. Lo que se pretende conseguir es que todas nuestras titulaciones den respuesta de la manera más eficaz y eficiente posible a las expectativas y necesidades actuales y futuras de nuestros grupos de interés, en el contexto en el que se encuentra nuestra universidad y se adapte rápidamente a los cambios del contexto.

Para ampliar el alcance del Sistema de Calidad, la Universidad de Deusto ha definido un Sistema de Gestión de Calidad Global. Este Sistema pretende poner en énfasis y lograr la satisfacción de la pieza esencial de nuestra universidad que es el estudiante. El objetivo es mejorar la satisfacción del estudiante desde su toma de contacto con el mundo universitario en los centros de enseñanza secundaria, hasta su inserción como egresado en el mercado laboral. El Sistema de Gestión de Calidad Global sigue las directrices del programa AUDIT de ANECA en las Facultades para las titulaciones de grado, máster y doctorado y de la norma ISO 9001:2015 para las Áreas Funcionales y Servicios de la Universidad relacionados más directamente con nuestro principal grupo de interés que son los estudiantes.

A continuación se resumen los criterios del programa AUDIT de ANECA:

- Cómo el Centro define su política y objetivos de calidad. El Centro debe consolidar una cultura de la calidad apoyada en una política y unos objetivos de calidad conocidos y accesibles públicamente, como compromiso con el aseguramiento de la calidad (ANECA, 2018, pág. 4).

- Cómo el Centro garantiza la calidad de sus programas formativos. El Centro debe contar con mecanismos que le permitan

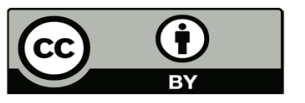

Licencia Creative Commons Atribución 4.0 Internacional (CC BY 4.0)

Revista Cátedra, 5(1), pp. 58-70, enero-junio 2022. e-ISSN: 2631-2875

https://doi.org/10.29166/catedra.v5i1.3451 
mantener y renovar su oferta formativa, desarrollando metodologías para el diseño, aprobación, control y revisión periódica (interna y externa), de los programas (ANECA, 2018, pág. 5).

- Cómo el Centro orienta sus enseñanzas a los estudiantes. El Centro debe dotarse de procedimientos que le permitan comprobar que las acciones que emprende tienen como finalidad fundamental favorecer el aprendizaje del estudiante y su acceso a las normas que regulan todas las fases del proceso formativo, desde su admisión, adquisición de competencias y habilidades, hasta la certificación de los resultados de aprendizaje adquiridos (ANECA, 2018, pág. 7).

- Cómo la Universidad y/o Centro garantiza y mejora la calidad de su personal académico. La Universidad y/o el Centro, debe contar con mecanismos que aseguren que el acceso, gestión, desarrollo y formación de su personal académico y de apoyo a la docencia, se realiza con las debidas garantías para que cumpla con las funciones que le son propias (ANECA, 2018, pág. 9).

- Cómo la Universidad y/o Centro gestiona y mejora sus recursos y servicios. La Universidad y/ o el Centro debe dotarse de mecanismos que le permitan diseñar, gestionar y mejorar los servicios de apoyo al alumnado, así como de los recursos humanos y materiales necesarios para facilitar un adecuado desarrollo de su aprendizaje (ANECA, 2018, pág. 10).

- Cómo el Centro analiza y tiene en cuenta la información de los resultados que obtienen los procesos del Sistema de Aseguramiento Interno de Calidad. El Centro debe dotarse de procedimientos que le permitan medir, analizar y utilizar los resultados generados por el Sistema de Aseguramiento Interno de Calidad, entre otros, del aprendizaje, de la satisfacción de los distintos grupos de interés y de la inserción laboral/ empleabilidad, para la toma de decisiones que conduzcan a una mejora de la calidad de las enseñanzas que imparte $\mathrm{y}$ del resto de actividades que realiza (ANECA, 2018, pág. 11).

- Cómo la Universidad publica la información sobre los títulos y otras actividades realizadas. La Universidad y/ o el Centro, debe dotarse de mecanismos que le permitan garantizar la publicación y difusión periódica de información fiable, actualizada y accesible, relativa a los títulos ofertadas y sus resultados, así como a otras actividades realizadas en aquel (ANECA, 2018, pág. 12).

- Cómo el Centro garantiza el mantenimiento y actualización del Sistema de Aseguramiento Interno de Calidad. El Centro debe disponer de una metodología de trabajo y de los recursos necesarios para diseñar e implantar un Sistema de Aseguramiento Interno de Calidad que ayude de manera eficaz al logro y mejora de sus resultados, y posibilite su evaluación externa con carácter periódico (ANECA, 2018, pág. 14).

\section{Sistemática de mejora continua. Pilar básico}

Uno de los pilares principales del Sistema de Aseguramiento de la Calidad consiste en la implantación de la sistemática de mejora continua siguiendo el Ciclo PDCA (Zaballa, 2000), tal y como se muestra en la Figura 1. Ciclo PDCA. 


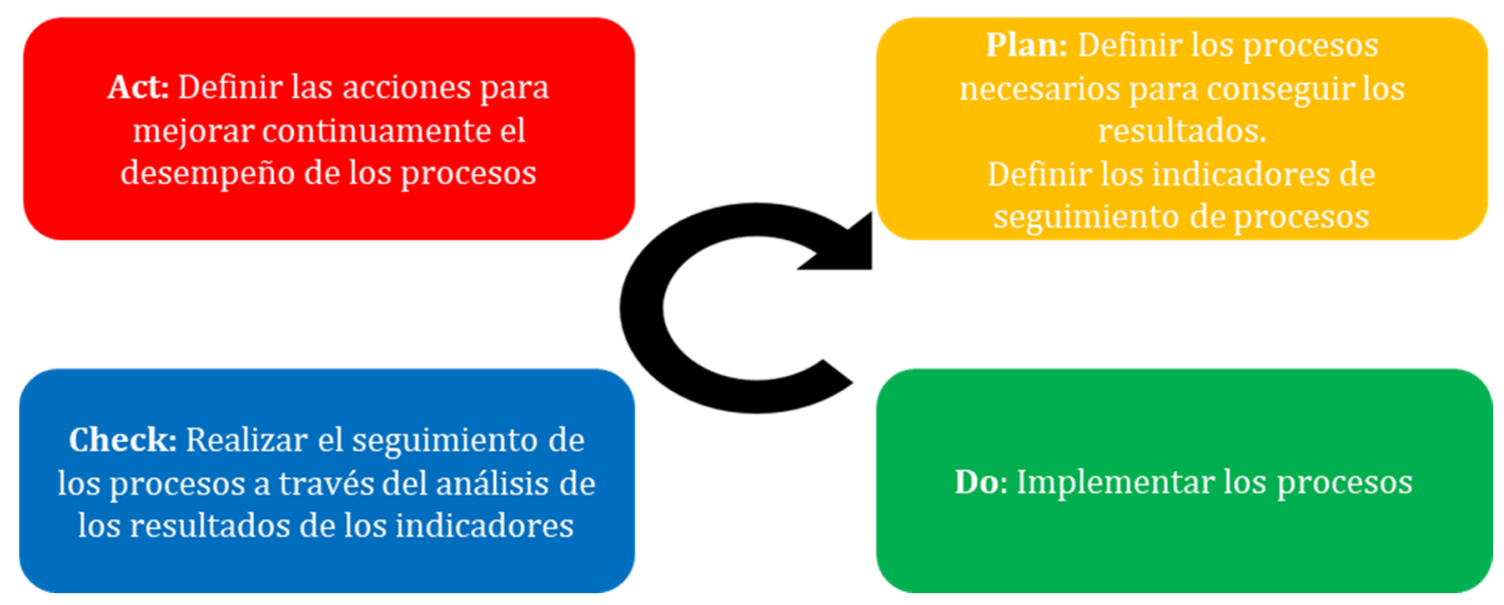

Figura 1. Ciclo PDCA

\subsection{Fase Plan}

La fase de planificación consiste en definir los procesos necesarios para cumplir las directrices del programa AUDIT de ANECA y conseguir a través de la implantación de los mismos los resultados planificados. Estos procesos se representan en un mapa de procesos, cuyo despliegue nos permite conseguir asegurar la mejora continua y sostenible de nuestra Universidad, nuestros Centros y nuestras titulaciones. Asimismo, nos facilita el cumplimiento de las directrices europeas y ministeriales respecto a la oficialidad de las titulaciones.

A continuación, se muestra una imagen del mapa de procesos de las Facultades de la Universidad de Deusto (Figura 2. Mapa de procesos).

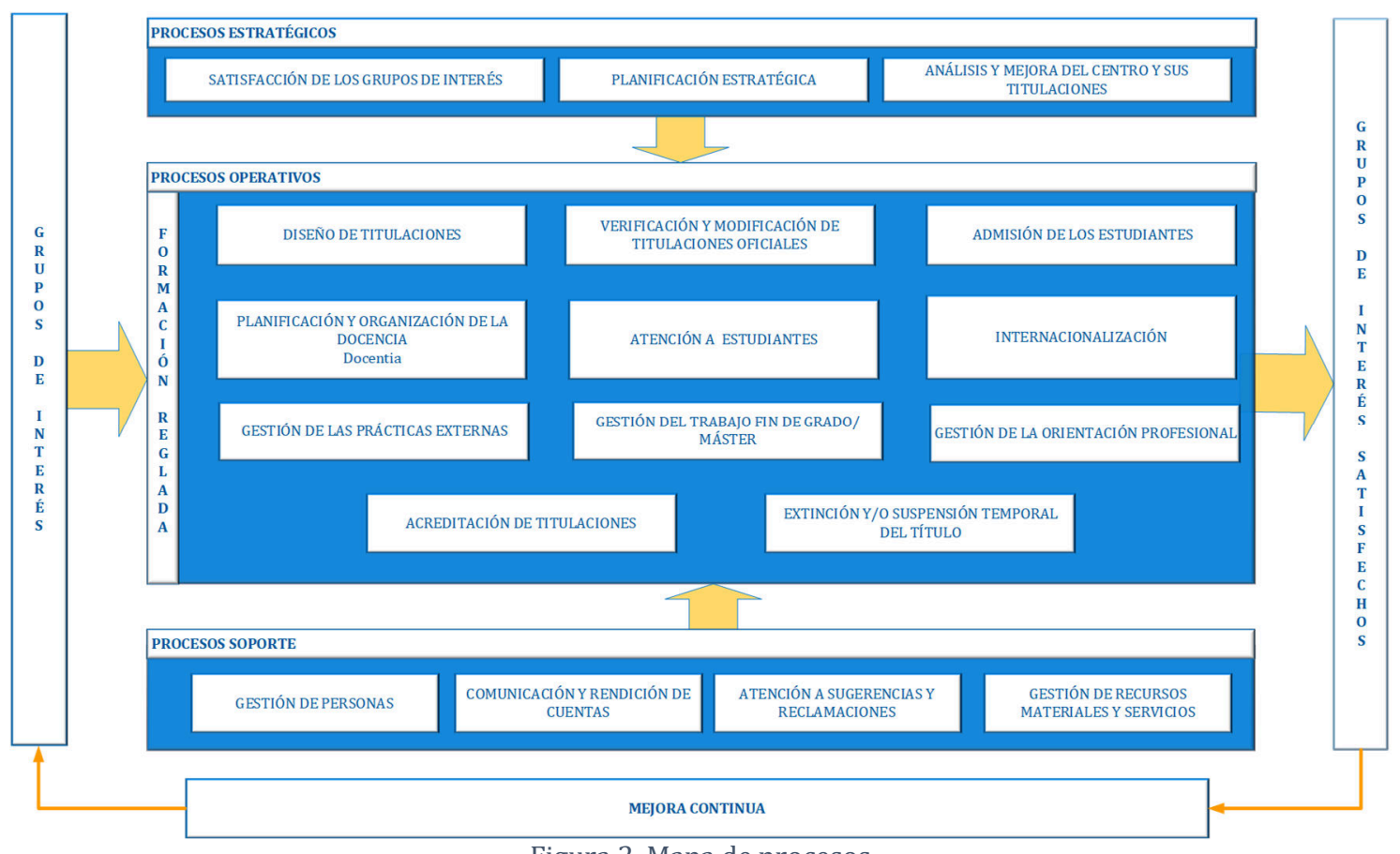

Figura 2. Mapa de procesos

Asimismo, en esta fase de planificación es muy importante definir los indicadores de seguimiento de los procesos y las metas a conseguir en cada indicador, para lograr los resultados planificados.

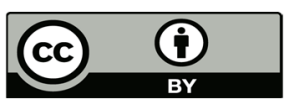




\subsection{Fase Do}

La fase de ejecución consiste en implantar los procesos planificados en la fase anterior.

\subsection{Fase Check}

La fase de verificación consiste en llevar a cabo el seguimiento de los procesos a través del análisis de los indicadores planificados para comprobar si se han alcanzado las metas definidas en la fase de planificación respecto a los indicadores de los procesos.

\subsection{Fase Act}

La fase de actuación consiste en definir las acciones necesarias para mejorar continuamente el desempeño de los procesos. En función de los resultados de la fase anterior, se identifican una serie de acciones de mejora, que se deben planificar para implementarlas.

\section{Ciclo de mejoramiento continuo del programa académico y de las facultades}

Los programas académicos de nuestra universidad, a lo largo de toda su vida académica, desde su diseño hasta su implantación, acreditación, reacreditación, caminan en una dirección única, que les obliga a una revisión permanente y como consecuencia de ello a la mejora continua de todos los programas académicos, de acuerdo a lo descrito en el Real Decreto 822/2021.

Esta sistemática de mejora continua se extiende a las facultades y a la universidad, a través de los procesos que están alineados con las directrices AUDIT. En la siguiente figura se representa el ciclo de mejora continua que siguen los programas académicos y las facultades en nuestra Universidad.

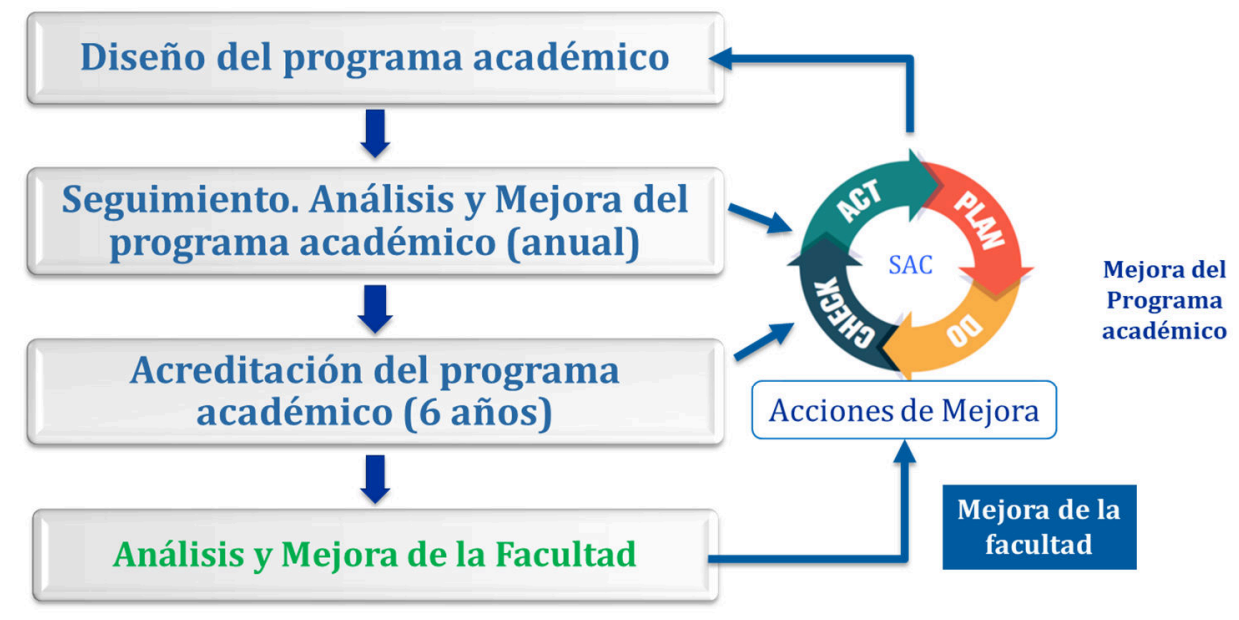

\section{Certificación del SAC (AUDIT, cada 5 años)}

Figura 3. Ciclo de mejoramiento continuo

\subsection{Diseño del programa académico}

En este proceso, se preparan las propuestas de los programas académicos universitarios oficiales diseñados en consonancia con el Espacio Europeo de Educación Superior. Se definen los siguientes apartados:

- Descripción del título.

- Justificación.

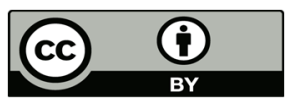


- Objetivos y competencias.

- Admisión de estudiantes.

- Planificación de las enseñanzas.

- Personal académico.

- Recursos previstos.

- Sistema de garantía de calidad.

- Calendario de implantación.

\subsection{Seguimiento, análisis y mejora del programa académico (anual)}

De acuerdo a lo descrito en las guías "Guía de Apoyo para el proceso de seguimiento de títulos oficiales de Grado y Máster" y "Guía de Apoyo para el proceso de seguimiento de títulos oficiales de Doctorado" (ANECA, 2016, 2020), anualmente se realiza el seguimiento de cada programa académico, es decir cómo va desarrollándose el programa anualmente en función de unos criterios.

Tal y como se explica en la publicación de Zaballa (2018), en la Universidad de Deusto tenemos implantado el proceso estratégico denominado "Análisis y mejora del Título", que lleva a cabo la Comisión de Calidad de cada Título. Dicha Comisión está constituida por los siguientes miembros:

- El responsable del título.

- Representantes del PDI.

- Representantes del PAS.

- Representantes de estudiantes.

- Representantes de estudiantes egresados.

- Representantes de los empleadores.

- Responsable de calidad de la Facultad.

- Representantes de la Unidad de Calidad de la Universidad de Deusto.

La misión de la Comisión de Calidad del Título consiste en una primera fase, en realizar anualmente un análisis exhaustivo de los resultados del proceso estratégico denominado "Satisfacción de los grupos de interés" (Zaballa y Liñero, 2017). Respecto a dicho proceso cabe destacar que a través del mismo se recoge la información sobre las necesidades y expectativas de nuestros grupos de interés.

La Universidad de Deusto entiende por grupo de interés a toda aquella persona, grupo o institución que tiene interés en la Universidad, en las enseñanzas o en los resultados obtenidos. Éstos incluyen al menos estudiantes, profesores, personal de administración y servicios, padres, administraciones públicas, empleadores y sociedad en general.

A través del proceso "Satisfacción de los grupos de interés" se establecen los mecanismos a aplicar para conocer, analizar y evaluar el grado de satisfacción de nuestros estudiantes, sus familias, egresados, empleadores, PDI y PAS, respecto al cumplimiento de sus necesidades y expectativas. De este modo, se obtendrá la información necesaria para aplicar la mejora continua a todas las actividades y procesos incluidos en el Sistema de Aseguramiento de la Calidad. Este proceso permite recoger y analizar la información relativa entre otros aspectos a:

- La calidad de la enseñanza y el profesorado.

- Las prácticas externas y los programas de movilidad.

- La inserción laboral de los graduados.

- La satisfacción con la formación recibida.

- La satisfacción de los distintos grupos de interés. 
Esta recogida de información se hace por varias vías: encuestas de medición de la satisfacción, focus group y reuniones con representantes de los estudiantes.

El proceso de satisfacción de los grupos de interés se inicia con la planificación de las mediciones que se llevarán a cabo en el curso académico. Para ello se establecen los objetivos de la medición, que permitirán definir las características y la amplitud de la misma. Además, se determinan también la población, la muestra invitada y la modalidad de medición: encuesta (personal, telefónica, correo, mail o fax), reclamaciones o sugerencias, contactos con los estudiantes, etc. Asimismo, es necesario establecer los criterios de análisis de los resultados, el responsable de realizar la medición y el plazo de realización de esta.

Basándose en la planificación establecida, la Unidad de Calidad de la Universidad diseña el material de apoyo que va a ser necesario para llevar a cabo las mediciones. Es importante tener en cuenta que dicho material se ha de adaptar al objetivo de la medición definido, al segmento de estudiantes objeto de la medición, al tiempo de respuesta que se establezca y al posterior tratamiento de los datos.

Una vez realizada la medición y recogidos sus resultados, la Unidad de calidad los analiza, de acuerdo a los criterios establecidos en la planificación inicial. Si no se obtiene el tamaño de muestra planificado, valorará en cada caso si conviene ampliar el periodo de medición o bien si se analizarán los resultados obtenidos hasta ese momento. Con los resultados obtenidos realiza un informe de medición de la satisfacción de los estudiantes, que de forma general incluye aspectos tales como la evaluación de resultados con datos cuantificables, una comparación retrospectiva de datos y los puntos fuertes y las áreas de mejora. La Unidad de Calidad envía este informe a los responsables de los títulos y del Centro.

Asimismo, la Comisión de Calidad del Título analiza los resultados del proceso soporte denominado "Atención a sugerencias y reclamaciones", de la información pública disponible, de la actividad docente y de los indicadores establecidos para todos nuestros procesos. Dicha comisión analiza también las acciones de mejora puestas en marcha, las tasas académicas obtenidas, los puntos débiles detectados y el informe de seguimiento del Título del curso académico anterior. Una vez hecho el análisis de toda la información relativa al Título, la Comisión de Calidad del Título obtendrá como resultado la información pública disponible revisada y actualizada, las recomendaciones señaladas por las agencias y el análisis de las tasas académicas. Además, se extraerán las fortalezas del Título en cuestión y las debilidades del mismo.

A partir de las fortalezas y debilidades encontradas en relación con el Título, la Comisión de Calidad del Título seguirá las pautas descritas en el proceso estratégico denominado "Acciones de Mejora" de nuestro Sistema de Gestión de Calidad. En dicho proceso, la Comisión de Calidad del Título deberá realizar un análisis riguroso para detectar las causas principales que han llevado a originar las debilidades surgidas en el Título. Una vez detectadas las causas, la Comisión de Calidad del Título deberá establecer las acciones de mejora más oportunas para subsanar las debilidades y someter a nuestros Títulos a un proceso de mejora continua.

Los pasos a llevar a cabo por los responsables de los Títulos consisten en hacer una descripción de la acción o acciones que se proponen para solucionar la debilidad en cuestión y nombrar al responsable o responsables de llevar a cabo la acción o acciones propuestas. Los responsables de los títulos tendrán que definir también el plazo o plazos que se fijarán como límite para la ejecución de dichas acciones y el parámetro mediante el cual se valorará la eficacia de la acción o acciones de mejora implantadas.

Asimismo, existe una Comisión de seguimiento de titulaciones a nivel de Universidad, cuyo principal objetivo es revisar los resultados del análisis y mejora de cada uno de nuestros

Licencia Creative Commons Atribución 4.0 Internacional (CC BY 4.0)

Revista Cátedra, 5(1), pp. 58-70, enero-junio 2022. e-ISSN:2631-2875

https://doi.org/10.29166/catedra.v5i1.3451 
programas formativos. Dicha revisión permite asegurar la rigurosidad de los resultados y el cumplimiento de los requisitos marcados internamente por la Universidad, así como de los requisitos externos exigidos por las agencias de calidad, otros organismos y grupos de interés.

La Comisión de seguimiento actúa una vez que se ha finalizado el proceso de Análisis y mejora de todos los Títulos y antes de que los resultados sean llevados a la Comisión de Calidad del Centro para su análisis. En el caso de que la Comisión de seguimiento observe algún tipo de incidencia, se lo comunicará al responsable de la Titulación para que la trate en la Comisión y actúe en consecuencia.

\subsection{Acreditación de titulación (6 años)}

La acreditación de titulaciones se desarrolla de acuerdo a lo descrito en la "Guía de Autoevaluación. Programa ACREDITA DOCTORADO" y en el “Documento Marco: Evaluación para la renovación de la acreditación de títulos oficiales de Grado y Máster Universitario" (ANECA, 2018, 2019). La acreditación tiene como objetivo comprobar si los resultados del título, entre los que se encuentran los resultados de aprendizaje, son adecuados y permiten garantizar la adecuada continuidad de la impartición del programa hasta la siguiente renovación de la acreditación (Figura 4. Criterios de evaluación del Programa Acredita). Se realiza cada seis años para los grados y másteres de manera obligatoria.

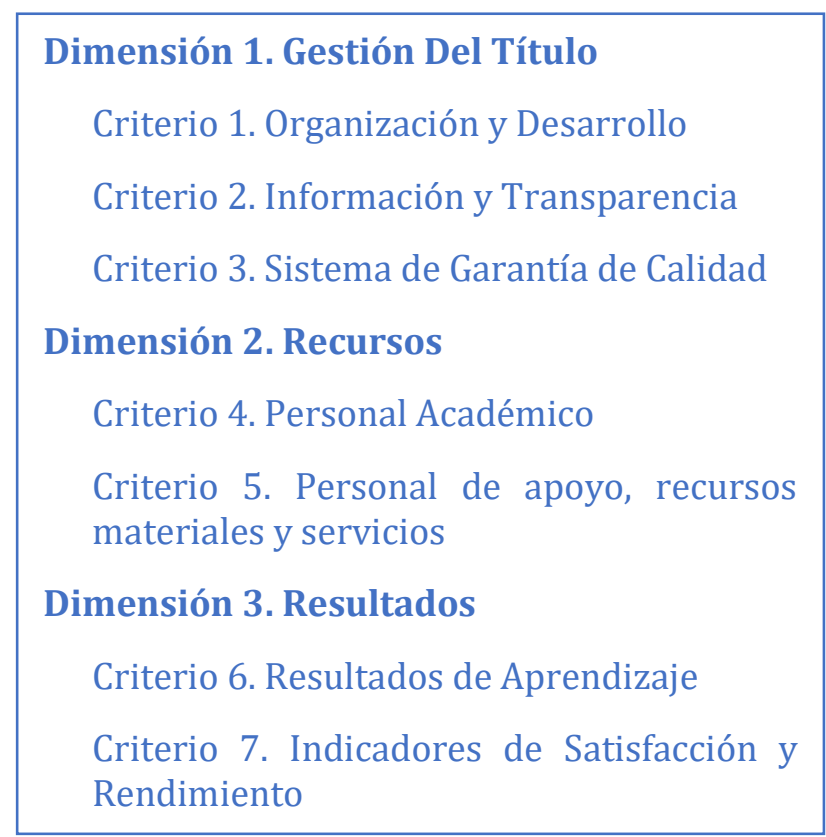

Figura 4: Criterios de evaluación del Programa Acredita

Para ello, la Comisión de Calidad del Título evalúa la consecución de los resultados de aprendizaje de la titulación, en función de lo planificado en la memoria verificada y asegurar así la mejora continua. Para ello, la Comisión de Calidad del Título analiza los siguientes aspectos:

- La implantación del plan de estudios y la organización del programa son coherentes con el perfil de competencias, objetivos y resultados de aprendizaje planificados.

- El título cuenta con mecanismos de coordinación docente que permiten tanto una adecuada asignación de carga de trabajo del estudiante como una adecuada planificación temporal, asegurando la adquisición de los resultados de aprendizaje. 
- Los estudiantes matriculados en el título, tienen acceso en el momento oportuno a la información relevante del plan de estudios y de los resultados de aprendizaje previstos.

- El Sistema de Aseguramiento de la Calidad implementado y revisado periódicamente garantiza la recogida y análisis continuo de información y de los resultados relevantes para la gestión eficaz del título, en especial los resultados de aprendizaje y la satisfacción de los grupos de interés.

- El personal de apoyo, los recursos materiales y los servicios puestos a disposición del desarrollo del título son los adecuados en función de la naturaleza, modalidad del título, número de estudiantes matriculados y competencias a adquirir por los mismos.

- En el caso de que el título contemple la realización de prácticas externas, estas se han planificado según lo previsto y son adecuadas para la adquisición de los resultados de aprendizaje del título.

- Las actividades formativas, sus metodologías docentes y los sistemas de evaluación empleados son adecuados y se ajustan razonablemente al objetivo de la adquisición de los resultados de aprendizaje previstos.

- Los resultados de aprendizaje alcanzados satisfacen los objetivos del programa formativo y se adecúan a su nivel en el MECES.

- El perfil de egreso definido (y su despliegue en competencias y resultados de aprendizaje en el plan de estudios) mantiene su relevancia y está actualizado según los requisitos de su ámbito académico, científico y profesional.

\subsection{Proceso de análisis y mejora de la facultad}

El proceso análisis y mejora de la Facultad sigue lo descrito en la "Guía de acreditación institucional" (ANECA, 2018) y tiene como objetivo incorporar la mejora continua al centro y a sus titulaciones, para lo que se realiza un análisis transversal del centro y de sus titulaciones. Para ello, existe una Comisión de Calidad del Centro en la que están representados todos los grupos de interés de la Facultad (Figura 5. Miembros de la Comisión de Calidad del Centro).

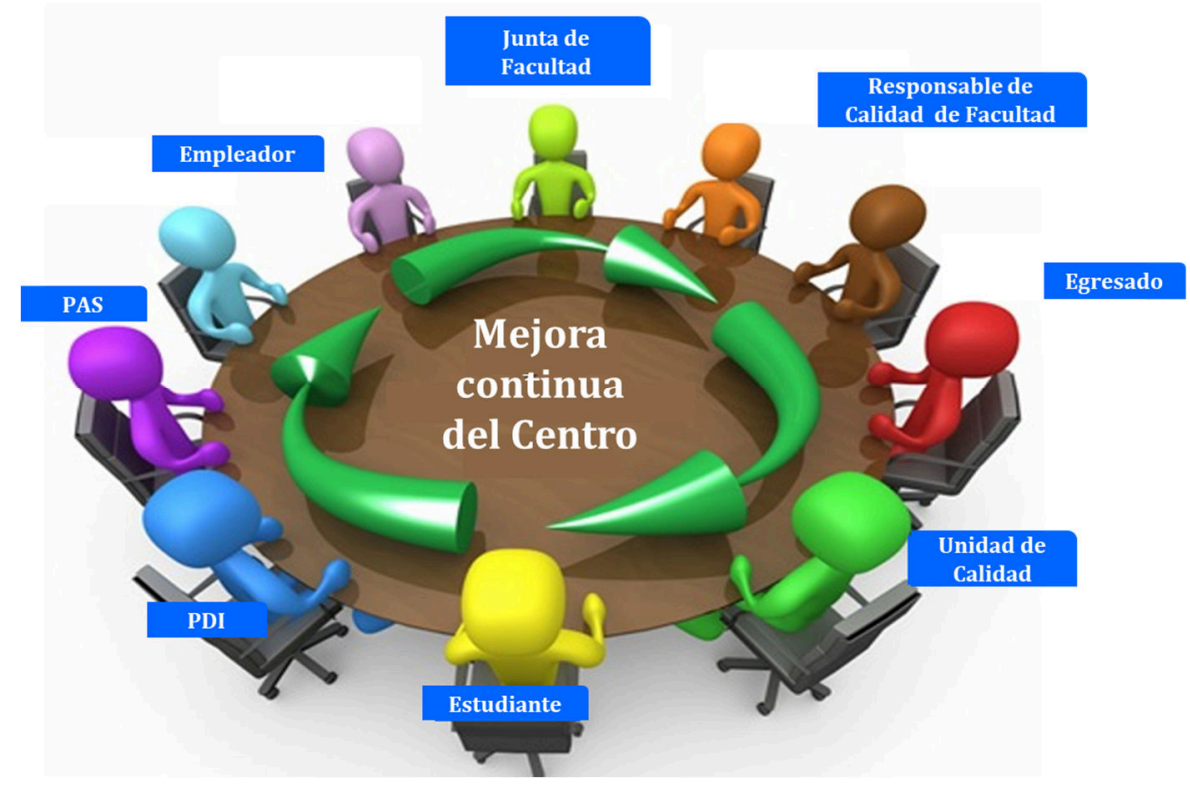

Figura 5: Miembros de la Comisión de Calidad del Centro

Dicha Comisión lleva a cabo el análisis de: 
- Adecuación y eficacia de la política y de los objetivos de calidad de la facultad fijados con base en esta.

- Grupos de Interés (sugerencias/reclamaciones y encuestas de satisfacción a los grupos de interés).

- Resultados de los procesos del Sistema de Aseguramiento de la Calidad de la Facultad.

- Estado de las acciones de mejora de la Facultad y de sus Títulos.

- Recomendaciones de las Agencias (feedback de Unibasq a los informes de Análisis y mejora del curso anterior).

Una vez hecho el análisis de la información citada anteriormente, la Comisión de Calidad del Centro obtendrá como resultados las fortalezas y debilidades de la Facultad y los resultados del proceso análisis y mejora de los títulos revisados y aprobados. La Comisión de Calidad del Centro también es responsable de añadir o eliminar aquella información del análisis de los títulos que considere necesaria.

\subsection{Proceso de acciones de mejora}

Las debilidades detectadas en los procesos anteriores constituyen el Plan de Mejora de cada programa académico y de la facultad. Para cada área de mejora identificada, se deben definir acciones concretas que permitan alcanzar los objetivos deseados. La gestión del Plan de mejora se lleva a cabo siguiendo lo descrito en el proceso estratégico "Acciones de mejora". Esto implica la planificación detallada de las acciones concretas a desarrollar, de los responsables asignados para llevar a cabo cada actividad planificada, de los plazos definidos para desarrollar las acciones y del parámetro de eficacia de la acción o acciones de mejora implantadas. Se lleva a cabo la ejecución de las acciones en los plazos establecidos y se hace el seguimiento de su implantación hasta la evaluación de la eficacia de las acciones realizadas. Así, aseguramos que las acciones de mejora se implantan, son eficaces para el propósito establecido y no se quedan en buenas intenciones.

\section{Beneficios del Sistema de Aseguramiento de la Calidad}

El Sistema de Aseguramiento de la Calidad nos ha ayudado a introducir de manera sostenible la cultura de calidad y de la mejora continua en la Universidad y se ha convertido en una herramienta eficaz para la implantación de manera sistemática de la mejora continua en los programas formativos, los Centros y la Universidad.

Respecto a los programas académicos nos ha permitido afianzar la sistemática de:

- Recogida de información de los procesos sobre el programa académico.

- Análisis de los resultados obtenidos para la toma de decisiones.

- Gestión del cuadro de mando integral del programa académico.

- Definición de acciones de mejora de los programas académicos para su adaptación a las necesidades del mercado laboral.

Asimismo, hemos logrado mejorar de manera sistemática mediante la evaluación y revisión los procesos académicos y de servicios:

- La calidad de la enseñanza impartida y del profesorado.

- De las prácticas externas y de la movilidad.

- De la satisfacción con la formación recibida y de la inserción laboral.

- Los resultados de aprendizaje.

- De la atención a sugerencias y reclamaciones y de la satisfacción de todos los colectivos implicados y la comunicación de los resultados.

- Por último, nos ha facilitado la acreditación de los programas académicos, ya que los procesos se van desarrollando durante todo el ciclo de vida de un programa

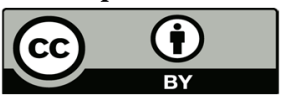

Licencia Creative Commons Atribución 4.0 Internacional (CC BY 4.0)

Revista Cátedra, 5(1), pp. 58-70, enero-junio 2022. e-ISSN: 2631-2875

https://doi.org/10.29166/catedra.v5i1.3451 
académico, lo que proporciona la recogida y análisis de los resultados del programa para la toma de decisiones y definición de los planes de mejora, todo ello de manera sistemática. Esta información está alineada con las necesidades para acreditar los programas académicos, de tal forma que cuando se tiene que acreditar, el Sistema de Aseguramiento de la Calidad nos ha ido proporcionando anualmente la información y los datos significativos para la definición de planes de mejora durante toda la vida de dicho programa. Los datos necesarios para un proceso de acreditación de un programa, los tenemos desde el primer año de implantación del programa a través de los procesos del Sistema de Aseguramiento de la Calidad. Es decir, no tenemos que empezar a recoger datos del programa cuando se decide acreditarlo.

\section{Conclusiones}

El Sistema de Aseguramiento de la Calidad es una herramienta útil para implantar la mejora continua en nuestras titulaciones, nuestras facultades, servicios y en la universidad a través de una gestión eficaz y sistemática de sus procesos. Está incorporado en la dinámica del día a día de la universidad y cada vez más interiorizado en los grupos de interés.

Debe ser flexible, adaptarse a los cambios y facilitar la constitución de universidades dinámicas, flexibles, innovadoras y de calidad. Facilita la acreditación de las titulaciones, la evaluación y mejora de la docencia, de las prácticas, la movilidad, la inserción laboral, el grado de satisfacción de nuestros grupos de interés, la implantación de la enseñanza basada en competencias, etc.

El Sistema de Aseguramiento de la Calidad nos permite lograr la satisfacción de los estudiantes desde su toma de contacto con el mundo universitario, hasta su inserción como egresados en el mercado laboral, y mejorar de manera sostenible el grado de satisfacción de nuestros grupos de interés, respecto a las necesidades y expectativas. Es importante y vital, que el Sistema de Aseguramiento de la Calidad se adapte a las necesidades de cada universidad y a cada contexto, respetando la autonomía de la universidad y sus lineamientos institucionales. 


\section{Bibliografía}

Agencia Nacional de Evaluación de la Calidad y Acreditación. (2016). Guía de Apoyo para el proceso de seguimiento de títulos oficiales de Grado y Máster. PROGRAMA MONITOR [Archivo pdf]. http://www.aneca.es/Programas-de-evaluacion/Evaluacion-detitulos/MONITOR/MONITOR-grado-master/Documentacion-y-herramientas

Agencia Nacional de Evaluación de la Calidad y Acreditación. (2018). Guía de acreditación institucional [Archivo pdf]. http://www.aneca.es/Programas-deevaluacion/Evaluacion-institucional/Acreditacion-institucional/Documentos-yherramientas

Agencia Nacional de Evaluación de la Calidad y Acreditación. (2018). Nuevo modelo del Programa AUDIT. ANECA [Archivo pdf]. http://www.aneca.es/Programas-deevaluacion/Evaluacion-institucional/AUDIT

Agencia Nacional de Evaluación de la Calidad y Acreditación. (2019). Documento Marco: Evaluación para la renovación de la acreditación de títulos oficiales de Grado y Máster Universitario. Programa ACREDITA [Archivo pdf]. http://www.aneca.es/content/download/12737/157930/file/acredita docmarco v6 190124.pdf

Agencia Nacional de Evaluación de la Calidad y Acreditación. (2020). Guía de Apoyo para el proceso de seguimiento de títulos oficiales de Doctorado. PROGRAMA MONITOR [Archivo pdf]. http://www.aneca.es/Programas-de-evaluacion/Evaluacion-detitulos/MONITOR/MONITOR-Doctorado/MONITOR-Doctorado-documentacion-yherramientas

Agencia Nacional de Evaluación de la Calidad y Acreditación. (2020). Guía de Autoevaluación. Programa ACREDITA DOCTORADO [Archivo pdf]. http://www.aneca.es/content/download/14069/174333/file/acredita doctorado Gu\%C3\%ADa\%20de\%20Autoevaluaci\%C3\%B3n julio2020.pdf

Real Decreto 822/2021, de 28 de septiembre, por el que se establece la organización de las enseñanzas universitarias y del procedimiento de aseguramiento de su calidad. 29 de septiembre de 2021, 119537 a 119578. https://www.boe.es/eli/es/rd/2021/09/28/822

Zaballa, G. (2018). Buenas prácticas en la gestión de calidad de las universidades, hacia la excelencia. Editorial Académica Española.

Zaballa, G. (2000). Modelo de calidad en Educación Goien. Ediciones Mensajero.

Zaballa, G. y Liñero, I. (2017). La satisfacción de nuestros grupos de interés, aspecto fundamental para la mejora de nuestras titulaciones. V Congreso Internacional de Docencia Universitaria.

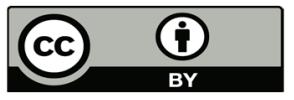




\section{Autores}

GLORIA ZABALLA-PÉREZ es doctora en Ingeniería Informática por la Universidad de Deusto, Máster en Ciencia y Tecnología de los Computadores y Máster en Gestión de Calidad. Ha recibido el Galardón a la Excelencia Educativa Edición 2020 y el Reconocimiento Honorífico Doctor Honoris Causa (Organización Internacional para la Inclusión y Calidad Educativa - OIICE). Sus principales líneas de investigación están relacionadas con la definición e implantación de sistemas de aseguramiento de la calidad sostenibles en las Instituciones de Educación Superior, la gestión de procesos académicos para asegurar su mejora continua, la evaluación de resultados de aprendizaje a través de los sistemas de aseguramiento de calidad de las universidades.

Actualmente es directora de la Unidad de Calidad de la Universidad de Deusto, y profesora titular de la Facultad de Ingeniería de la Universidad de Deusto. También es profesora docente colaboradora de la Universidad Oberta de Cataluña en el Máster universitario de Evaluación y Gestión de la Calidad en la Educación Superior. Posee una amplia experiencia en evaluación/acreditación de carreras, facultades y centros, así como en la certificación de la implantación de sistemas de calidad en las facultades y universidades a nivel nacional e internacional en las diferentes Agencias de Calidad universitarias y organismos de Acreditación. Posee numerosas publicaciones y participaciones en congresos, y conferencias a nivel nacional e internacional. 